Pacific Journal of Mathematics

THE LATTICE OF CONGRUENCES ON AN INVERSE 


\title{
THE LATTICE OF CONGRUENCES ON AN INVERSE SEMIGROUP
}

\author{
DAVID G. GREeN
}

\begin{abstract}
The symmetry between four congruences which have figured in the literature on inverse semigroups is discussed in terms of kernels and restrictions to idempotents (kernel meaning the set of elements equivalent to idempotents). A characterization of arbitrary inverse semigroup congruences is developed from this symmetry.
\end{abstract}

1. Introduction. Inverse semigroup congruences were first studied in terms of kernel normal systems. Preston [11] defined a kernel normal system over an inverse semigroup $S$ to be any collection of inverse subsemigroups of $S$ satisfying certain conditions. $\mathrm{He}$ showed [11, Theorem 1; also 1, Theorem 7.48] that if $\mathscr{A}=\left\{A_{i}: i \in I\right\}$ is a kernel normal system over $S$, then $\mathscr{A}$ uniquely determines a congruence $\rho_{\mathscr{A}}$ on $S$ where $\rho_{\mathscr{S}}=\left\{(a, b) \in S \times S: a a^{-1}, b b^{-1}, a b^{-1} \in A_{i}\right.$, for some $i \in I\}$; and, conversely, that if $\rho$ is some congruence on $S$, then the set of equivalence classes of $\rho$ containing idempotents of $S$ forms a kernel normal system $\mathscr{A}$ and $\rho=\rho_{\mathscr{A}}$. Hence if $\alpha$ and $\beta$ are congruences on $S$, to show that $\alpha$ is contained by $\beta$ it suffices to show that if $(a, e) \in \alpha$, with $e$ idempotent, then $(a, e) \in \beta$.

In what follows we shall concern ourselves with kernels of congruences instead of kernel normal systems. The kernel (ker $\rho$ ) of a congruence $\rho$ on an inverse semigroup $S$ will be taken to mean the set of all elements of $S$ identified with idempotents by $\rho$. Ker $\rho$ is thus the union of the classes in the kernel normal system of $\rho$.

In [14], Reilly and Scheiblich considered the restriction to idempotents of inverse semigroup congruences. They classified congruences in terms of restrictions to idempotents and characterized minimum and maximum congruences having any given restriction. In [15], Scheiblich has extended this approach by showing that each congruence on an inverse semigroup can be uniquely characterized in terms of its restriction to idempotents together with its kernel. We shall eventually obtain Scheiblich's result again here; but by a different route which fills in details that clarify the situation.

We shall assume throughout (unless otherwise stated) that we are dealing with a fixed, but otherwise arbitrary, inverse semigroup $S$ with semilattice of idempotents $E$ (or $E(S)$ when necessary). The expression $\alpha(T)$ will be used to emphasize that $\alpha$ is a relation on the inverse semigroup $T ; \alpha \mid R$ will denote the restriction of $\alpha$ to a subset $R$ of $T$. Finally, $L(S)$ will represent the lattice of congruences on $S$. 


\section{Special congruences.}

2.1. Within the lattice of congruences on any inverse semigroup, there is a number of congruences which, owing to the nature of inverse semigroups, are readily distinguishable. The trivial congruences $c$ (which identifies elements with themselves only) and $\omega$ (which identifies together all elements) can immediately be singled out. We shall consider four others:

(a) A congruence $\rho$ on $S$ will be called a semilattice congruence if and only if $S / \rho$ is a semilattice. The maximum semilattice congruence is thus $\omega$. Now, $a \rho$ is idempotent in $S / \rho$ if and only if $a \rho=a a^{-1} \rho$ [1, Lemma 7.34]. Also, for Green's relation $\mathscr{R},\left(a, a a^{-1}\right) \in \mathscr{R}$, for any $a \in S$; and $(a, b) \in \mathscr{R}$ if and only if $a a^{-1}=b b^{-1}$. So $\rho$ is a semilattice congruence just in case it contains $\mathscr{R}$. The minimum semilattice congruence (here denoted by $\lambda$ ) is thus the least congruence containing $\mathscr{R}$; that is, from Preston's characterization [1, Lemma 10.3] of the least congruence containing a given equivalence, $\lambda=\mathscr{R}^{*}=$ $\left\{(x, y) \in S \times S: x=s u t, y=s v t \text { for some }(u, v) \in \mathscr{R}, s, t \in S^{1}\right\}^{t}$.

(b) Idempotent-separating congruences on $S$ are those congruences under which no two idempotents of $S$ are equivalent. The least of these is $\iota$; the maximum one Howie [3] characterized as $\mu$, where $\mu=\left\{(x, y) \in S \times S: x^{-1} e x=y^{-1} e y\right.$, for every $\left.e \in E\right\}$.

(c) A congruence $\rho$ on $S$ is called a group congruence just in case $S / \rho$ is a group. The greatest group congruence is thus $\omega$. In [8], Munn showed that $\rho$ is a group congruence if and only if it contains the least group congruence $\sigma$, where $\sigma=\{(x, y) \in S \times S: e x=$ $e y$, for some $e \in E\}$.

(d) Idempotent-determined congruences on $S$ are those congruences under which no idempotents of $S$ are equivalent to nonidempotent elements. The minimum idempotent-determined congruence must be $\iota$. It has been shown [2, Prop. 1] that a greatest idempotentdetermined congruence exists; we denote it by $\tau$. The relation $(E \times E) \cup$ $(S \backslash E \times S \backslash E)$ contains all idempotent-determined congruences; so, from Preston's expression [1, Lemma 10.3] for the largest congruence contained in an equivalence, the following characterization is almost immediate.

Proposition 2.1. The maximum idempotent-determined congruence on an inverse semigroup $S$ is the relation $\tau(S)$, where $\tau(S)=$ $\left\{(x, y) \in S \times S:\right.$ for all $u, v \in S^{1}, u x v \in E$ if and only if $\left.u y v \in E\right\}$.

2.2. We shall now examine the symmetry between the congruences $\lambda, \mu, \sigma$, and $\tau$.

Since $L(S)$ is a complete lattice, all four classes of congruence 
introduced above form complete sublattices of $L(S)$. It has been shown [1, Theorem 7.58] that the set of all idempotent-separating congruences on $S$ forms a principal ideal of $L(S)$; the same has been shown [2, Prop. 1] for idempotent-determined congruences. In the last section, we saw that only semilattice congruences contain $\lambda$ and that only group congruences contain $\sigma$. Hence these last two classes of congruence form principal filters of $L(S)$ (filter is dual to ideal).

Since congruences on groups correspond to normal subgroups and because the normal subgroups of a group form a modular lattice, the lattice of group congruences on $S$ is modular. The lattice of idempotent-separating congruences on $S$ is modular also [1, Theorem 7.58]. There are semilattices (for example the five-element, nonmodular lattice) whose lattices of congruences are nonmodular. Also, if $S$ is a semilattice, every congruence on $S$ both contains $\lambda$ and is contained in $\tau$. Thus neither the lattice of semilattice congruences nor the lattice of idempotent-determined congruences is modular in general.

The following two results help us to picture the way our special congruences relate to each other.

Proposition 2.2. On any inverse semigroup $S, \mu(S)$ is the greatest congruence contained in the Green's relation $\mathscr{R}$; while $\lambda(S)$ is the least congruence containing $\mathscr{R}$.

The first assertion is true because $\mathscr{R}$ separates idempotents and because $\mu$ is the greatest congruence contained in Green's relation $\mathscr{H}$ (see [9]). We proved the second assertion above.

Proposition 2.3. Let $\mathscr{F}=\left\{(a, b) \in S \times S: a^{-1} b \in E\right\}$. Then $\tau(S)$ is the greatest congruence contained in $\mathscr{F}$ and $\sigma(S)$ is the least congruence containing $\mathscr{F}$.

Proof. It follows from the proof of [2, Lemma 1] that a congruence is idempotent-determined if and only if it is contained in $\mathscr{F}$. The first assertion is immediate from this. If $(a, b) \in \mathscr{F}$, then $a^{-1} b \in E$. This implies that $a^{-1} \sigma=(b \sigma)^{-1}$ and hence that $a \sigma=b \sigma$. It follows that $\mathscr{F}$ is contained in $\sigma$. If $a \sigma b$, where $a, b \in S$, then (for some $e \in E) a \mathscr{F} e a, e a=e b$ and $e b \mathscr{F} b$; hence $\sigma=\mathscr{F}^{*}$.

REMARK 2.4. Both results above still hold if $\mathscr{R}$ is replaced by $\mathscr{L}$ and $\mathscr{F}$ by $\mathscr{B}$, where $\mathscr{B}=\left\{(a, b): a b^{-1} \in E\right\}$.

For any two elements $a$ and $b$ of $S, a \mathscr{F}\left(b b^{-1}\right) a,\left(b b^{-1}\right) a \mathscr{R}\left(a a^{-1}\right) b$, and $\left(a a^{-1}\right) b \mathscr{F} b$. This shows that $\omega$ is the least equivalence containing both $\mathscr{R}$ and $\mathscr{F}$ (note that $\mathscr{F}$ is not transitive in general). Con- 
versely, from [2, Lemma 1], if $a \mathscr{F} b$ and $a \mathscr{R} b$, then $a=b$. This means that $\mathscr{R} \cap \mathscr{F}=\iota$. It follows that in $L(S), \lambda \vee \sigma=\omega$ and $\mu \cap \tau=\iota$.

Figure 1, then, gives a partial picture of $L(S)$. Note that the elements shown do not form a sublattice of $L(S)$ in general.

2.3. The kernel of any congruence $\rho$, say, on $S$ is $S$ itself just where $\rho$ is a semilattice congruence; so $\lambda$ is the least congruence having $S$ as kernel. Similarly, group congruences are just those congruences whose restriction to $E$ is $\omega(E)$; so $\sigma$ is the least congruence having $\omega(E)$ as its restriction to $E$. By definition, $\mu$ is the greatest congruence whose restriction to $E$ is $\iota(E)$ and $\tau$ is the greatest congruence whose kernel is $E$. We can also state

Proposition 2.5. Let $\rho$ and $\xi$ be congruences on $S$.

(i) If $\operatorname{ker} \xi=\operatorname{ker} \rho$ and $\rho$ is a group congruence, then $\xi \leqq \rho$.

(ii) If $\operatorname{ker} \xi=\operatorname{ker} \rho$ and $\rho$ is idempotent-separating, then $\xi \supseteqq \rho$.

(iii) If $\xi|E=\rho| E$ and $\rho$ is a semilattice congruence, then $\xi \leqq \rho$.

(iv) If $\xi|E=\rho| E$ and $\rho$ is idempotent-determined, then $\xi \supseteqq \rho$.

Proof. (i) Let $(a, e) \in \xi$, with $e$ idempotent. Then $a \in \operatorname{ker} \rho$; since $\operatorname{ker} \rho=\operatorname{ker} \xi$ and $a \in \operatorname{ker} \xi$. But, because $\rho$ is a group congruence, its kernel normal system consists of just the one class $\operatorname{ker} \rho$. So $(a, e) \in \rho$.

(ii) Let $(a, e) \in \rho$ with $e$ idempotent. Since $\operatorname{ker} \rho=\operatorname{ker} \xi, a \epsilon$ ker $\xi$ : so that $\left(a, a a^{-1}\right) \in \xi$. Since $\rho$ is idempotent-separating, $e=a a^{-1}$. Thus $(a, e) \in \xi$.

(iii) Let $(a, e) \in \xi$ with $e$ idempotent; then $\left(a a^{-1}, e\right) \in \xi$. Since $\xi|E=\rho| E,\left(a a^{-1}, e\right) \in \rho$ also. Because $\rho$ is a semilattice congruence, $\left(a, a a^{-1}\right) \in \rho$; and hence $(a, e) \in \rho$.

(iv) Let $(a, e) \in \xi$ with $e$ idempotent. Then $a \in E$, since $\xi$ is idempotent-determined, and hence $(a, e) \in \xi|E=\rho| E$. Thus $(a, e) \in \rho$.

2.4. We shall pursue the above ideas further in the next section.

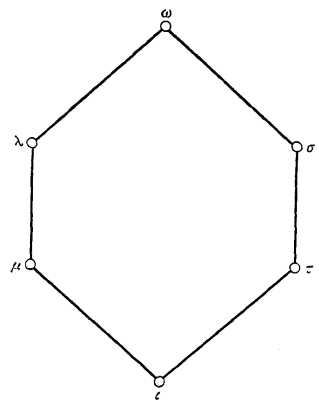

Figure 1. 
Some examples will show how our special congruences vary on particular kinds of inverse semigroups.

(1) $S$ is a semilattice if and only if $\lambda=\mu=\iota$ and $\omega=\sigma=\tau$.

(2) $S$ is a group if and only if $\omega=\lambda=\mu$ and $\sigma=\tau=\iota$.

(3) If $S$ is a semilattice of groups, the relation $\mathscr{R}$ is compatible; hence $\mu=\lambda$. Conversely, if these two congruences are equal on $S$, then, because $\operatorname{ker} \lambda=S$ and $\operatorname{ker} \mu$ is always a semilattice of groups, $S$ itself must be a semilattice of groups.

(4) Let $\mathscr{I}_{X}$ denote the symmetric inverse semigroup on a set $X$. Scheiblich [16] has shown that on $\mathscr{F}_{X}: \mu=\tau=\iota ; \sigma=\omega ; \lambda=\omega$ if $X$ is empty or infinite; and $\mathscr{I}_{X} / \lambda$ is the two-element semilattice if $X$ is finite and nonempty.

(5) An inverse semigroup on which $\mu=\ell$ is called fundamental. In [10], Munn showed that any fundamental inverse semigroup $S$ can be embedded in the semigroup $T_{E}$, which consists of all isomorphisms between principal ideals of $E$. In particular, $T_{E}$ itself is fundamental.

(6) Any inverse semigroup on which $\tau=\sigma$ is called proper. McAlister [4], [5], [6] has shown that proper inverse semigroups are precisely those inverse semigroups isomorphic to P-semigroups (whose structure is described explicitly in terms of certain groups, semilattices, and partially-ordered sets) and, moreover, that every inverse semigroup is an idempotent-separating, homomorphic image of some $P$-semigroup.

(7) McFadden and McAlister [7] showed that the free inverse semigroup $I_{X}$, on a set $X$, is proper. Reilly [12, Lemma 1.3] showed that $\mathscr{H}$ is trivial for $I_{X}$; so $I_{X}$ is fundamental also. However, since there exists a semilattice $E$ (say) of cardinality $\alpha$, for each nonzero $\alpha$, and since each $E$ is a homomorphic image of $I_{E}, \lambda\left(I_{E}\right)$ is never trivial.

(8) Let $\alpha$ denote any one of the four congruences $\lambda, \mu, \sigma$, and $\tau$. If $S$ and $T$ are inverse semigroups and $S \times T$ is given the usual product (componentwise multiplication), then, from the definition or characterization in each case: $(s, t) \alpha(S \times T)\left(s^{\prime}, t^{\prime}\right)$ if and only if $s$ $\alpha(S) s^{\prime}$ and $t \alpha(T) t^{\prime}$.

It follows that if $E$ is a semilattice, $G$ a group, and $\mathscr{F}_{X}$ the symmetric inverse semigroup on a set $X$ (and all three are nontrivial), then, by (1), (2), and (4) above, $E \times G \times \mathscr{I}_{X}$ is an inverse semigroup on which $\lambda, \mu, \sigma$, and $\tau$ are all nontrivial and no two of them equal.

(9) $S$ is called congruence-free if there are no congruences on $S$ distinct from $\iota$ and $\omega$ (see [10]). In this case $S$ must have one (or more) of three forms:

(a) $\tau=\sigma=\omega$ (equivalently $\iota=\mu=\lambda$ ). Since $\lambda=\iota, S$ must be a semilattice. Let $[e]$ denote the principal ideal containing $e$, for any 
$e$ in $S$. Then the relation $\iota(S) \cup \omega([e])$, which simply collapses $[e]$, is a congruence on $S$ and must therefore be $\iota(S)$ or $\omega(S)$. Hence either $[e]=\{e\}$ or $[e]=S$, for every $e$ in $S$. This is impossible if $S$ has more than two elements; so $S$ must be either the one- or two-element semilattice.

(b) $\iota=\tau=\sigma$ (equivalently $\mu=\lambda=\omega$ ). Since $\sigma=\iota, S$ must be a group. Since congruences on groups correspond to normal subgroups, it follows that $S$ must be a simple group.

(c) $\iota=\tau, \sigma=\omega$ (equivalently $\iota=\mu, \lambda=\omega$ ). Munn [10] showed that $S$ has this form if and only if it is isomorphic to a full subsemigroup of $T_{E}^{*}$, which consists of those elements of $T_{E}$ that contain identity maps of principal ideals of $E$.

3. The general lattice. There are two strands to our analysis of the lattice $L(S)$. First, we adapt and summarize some of Reilly and Scheiblich's main results from [14]. These results classify congruences by their restrictions to idempotents. We then derive similar results for kernels. The two threads will be tied together finally, giving alternatives to Scheiblich's results in [15].

3.1. A congruence $\pi$ on $E$ will be called normal if and only if, for any $e, f \in E$ and every $a \in S$, exf implies $a^{-1} e a \pi a^{-1} f a$. The relevance of normal congruences is shown by

THEOREM 3.1. The restriction to $E$ of any congruence on $S$ is a normal congruence on $E$. Conversely, for every normal congruence $\pi$ on $E$, there exist minimum and maximum congruences on $S$ whose restriction to $E$ is $\pi$. These are (respectively) $\sigma_{\pi}$ and $\mu_{\pi}$, where $\sigma_{\pi}=\left\{(a, b) \in S \times S: a a^{-1} \pi b b^{-1}\right.$ and $e a=e b$ for some e $\left.\pi a a^{-1}\right\}$; and $\mu_{\pi}=$ $\left\{(a, b) \in S \times S:\right.$ for all $e, f \in E$, e $\pi f$ implies aea $\left.a^{-1} \pi b f b^{-1}\right\}$.

The first assertion is easily proved: any congruence is a compatible relation. The remainder is a restatement of [14, Theorem 4.2]. We have spoken of "normal congruences on $E$ " rather than "normal partitions of $E$ " (see [14, Def. 4.1]) since we shall consider restrictions of given congruences to $E$. The correspondence between the two terms should be clear.

A relation $\theta$ can be defined on $L(S)$ by putting $\left(\rho_{1}, \rho_{2}\right) \in \theta$ if and only if $\rho_{1}\left|E=\rho_{2}\right| E$. This has the following properties.

TheOREM 3.2 ([14, Theorem 5.1]). Let $S$ be an inverse semigroup and define the relation $\theta$ on $L(S)$ as above. Then

(i ) $\theta$ is a congruence on $L(S)$;

(ii) each $\theta$-class is a complete, modular sublattice of $L(S)$ (hence 
with greatest and least element).

The sublattice of idempotent-separating congruences on $S$ and the sublattice of group congruences on $S$ fall naturally into place as the $\theta$-classes containing (respectively) $\iota(S)$ and $\omega(S)$. Also, $\mu_{\iota(E)}=\mu(S)$ and $\sigma_{\omega(E)}=\sigma(S)$, thus motivating our notation above (see also [13] for this notation).

3.2. We now classify congruences by their kernels. Let $R$ denote any full inverse subsemigroup of $S$; that is, $R$ contains $E(S)$. From Preston's characterization [1, Lemma 10.3], the greatest congruence contained in $(R \times R) \cup(S \backslash R) \times(S \backslash R)$ is $\gamma$, where $\gamma=\{(a, b) \in S \times S$ : $x a y \in R$ if and only if $x b y \in R$, for all $\left.x, y \in S^{1}\right\}$. Any congruence contained by $\gamma$ has its kernel contained in $R$. If $R$ is the kernel of a congruence, then $\operatorname{ker} \gamma=R$ and $\gamma=\tau_{R}$, where $\tau_{R}$ denotes the greatest congruence whose kernel is $R$. Conversely, any congruence whose kernel contains $R$ must identify each element $x$ of $R$ with $x x^{-1}$. Hence the least congruence whose kernel contains $R$ is the least congruence containing $\mathscr{R}(R) \cup \iota(S)$. From [1, Lemma 10.3], this is the relation $\delta$, where $\delta=\left\{(a, b) \in S \times S\right.$ : for some $r, s \in R, x, y \in S^{1}, r r^{-1}=$ $s s^{-1}$ and $\left.x r y=a, x s y=b\right\}^{t}$. If $R$ is the kernel of a congruence, then $\delta=\lambda_{R}$, where $\lambda_{R}$ denotes the minimum congruence having $R$ as kernel.

DEFINITION. An inverse subsemigroup $R$ of an inverse semigroup $S$ is called normal in $S$ if and only if

(a) $R$ is a full inverse subsemigroup of $S$; and

(b) for any $x, y$ in $S, r \in R$ and $x y \in R$ imply $x r y \in R$.

To ensure that the definition is reasonable, we must check that our conditions reduce to those for normal subgroups when both $R$ and $S$ are groups. In such circumstances the first condition becomes trivial. Now, if $R$ and $S$ are groups, $R$ is a normal subgroup of $S$ just in case it satisfies the equivalent defining properties: (i) $x R=R x$, for each $x \in S$; and (ii) $x r x^{-1} \in R$, for each $r \in R$ and each $x \in S$. A simple check shows that (b) implies (ii) and (i) implies (b).

The next result, an analogue of Theorem 3.1, explains the role of normal inverse subsemigroups.

THEOREM 3.3. For any congruence $\alpha$ on an inverse semigroup $S$, $\operatorname{ker} \alpha$ is normal in $S$. Conversely, if $R$ is normal in $S$, then $\tau_{R}$ and $\lambda_{R}$ are the greatest and least congruences (respectively) having $R$ as kernel.

Proof. For any congruence $\alpha, \operatorname{ker} \alpha$ is necessarily a full subsemigroup of $S$. Let $r$ be any element of $\operatorname{ker} \alpha$. For any $x, y$ in $S$, 
if $x y \in \operatorname{ker} \alpha$, then $x y\left(y^{-1} r r^{-1} y\right)$ belongs to ker $\alpha$; so that $x r r^{-1} y$ belongs to $\operatorname{ker} \alpha$ too. As $x r y$ is equivalent to $x r r^{-1} y$ under $\alpha$, it follows that xry must be an element of $\operatorname{ker} \alpha$. This ensures that $\operatorname{ker} \alpha$ is normal.

Suppose that $R$ is normal in $S$. For the congruence $\gamma$ defined above, $\operatorname{ker} \gamma$ is contained in $R$. If $x r y$ is an element of $R$, where $x, y \in S^{1}$ and $r \in R$, then $x r r^{-1} y$ is also an element of $R$ (because $r^{-1} \in R$ and $R$ is normal). Similarly, the converse is true; so $r \gamma r r^{-1}$ and hence $R=\operatorname{ker} \gamma$. Because $R$ is the kernel of some congruence on $S$, it follows immediately that $\tau_{R}$ and $\lambda_{R}$, as defined above, are the maximum and minimum congruences (respectively) having $R$ as kernel.

From the above result, we deduce that the sublattice of idempotent-determined congruences and the sublattice of semilattice congruences are the special classes of congruence having $E$ and $S$, respectively, as kernel. It follows that $\tau_{E}=\tau(S)$ and $\lambda_{S}=\lambda(S)$, which again motivates our notation.

THeOREM 3.4. The set of all normal inverse subsemigroups of an inverse semigroup $S$ forms a complete lattice $N(S)$ with greatest and least element. The map $\alpha \mapsto \operatorname{ker} \alpha$ is a $\cap$-semilattice homomorphism of $L(S)$ onto $N(S)$.

Proof. Let $M$ denote the intersection of an arbitrary set $P$ of normal inverse subsemigroups of $S . M$ is a full inverse subsemigroup of $S$. If $m$ belongs to $M$, then $m$ belongs to each element of $P$; whence, for all $x, y$ in $S, x y$ belongs to $M$ implies that $x m y$ belongs to $M$; so $M$ is normal in $S$. Also, as $M$ is the greatest set contained in every element of $P$, it must be the greatest lower bound of $P$ in $N(S)$. Conversely, since $S$ itself is normal in $S$, there are normal inverse subsemigroups which contain every element of $P$. By the same argument as before, the intersection of these is normal in $S$ and, since this intersection contains every element of $P$, it must be the least upper bound of $P$ in $N(S)$. Hence $N(S)$ is a complete lattice. As we have seen, $S$ and $E$ are both normal in $S$ : they are necessarily the greatest and least elements of $N(S)$.

Finally, for any elements $\alpha, \beta$ in $L(S), \operatorname{ker}(\alpha \cap \beta)=\left\{x: x \alpha x x^{-1}\right.$ and $\left.x \beta x x^{-1}\right\}=\operatorname{ker} \alpha \cap \operatorname{ker} \beta$. This proves the second assertion.

3.3. We can now combine our two approaches to decomposing $L(S)$. For any congruence $\alpha$ on $S$, we can relate the lattice $L(S / \alpha)$. to $L(S)$. If $\beta \in L(S)$ and $\beta \supseteqq \alpha$, define the relation $\beta / \alpha$ on $S / \alpha$ by:

$$
(x \alpha, y \alpha) \in \beta / \alpha \text { if and only if }(x, y) \in \beta \text {. }
$$

This relation is a congruence on $S / \alpha$ (see $[14, \S 3]$ ). The following well-known result (see [14, §3], for example) is true for virtually any 
algebraic structure.

Lemma 3.5. Let $\alpha$ denote any congruence on $S$. The map $\beta \mapsto$ $\beta / \alpha$ is an isomorphism from the lattice of those congruences on $S$ that contain $\alpha$ onto $L(S / \alpha)$, the lattice of all congruences on $S / \alpha$.

This result leads to formulae for computing particular congruences on $S / \alpha$.

Proposition 3.6. For any congruence $\alpha$ on an inverse semigroup $S:$

(i ) $\lambda(S / \alpha)=(\lambda(S) \vee \alpha) / \alpha$;

(ii) $\mu(S / \alpha)=\mu_{\alpha \mid E} / \alpha$;

(iii) $\sigma(S / \alpha)=(\sigma(S) \vee \alpha) / \alpha$;

(iv) $\tau(S / \alpha)=\tau_{\operatorname{ker} \alpha} / \alpha$.

Proof. (i ) If $\beta \in L(S)$, then $S /(\beta \vee \alpha) \cong(S / \alpha) /((\beta \vee \alpha) / \alpha)$ by Lemma 3.5. Thus $(\beta \vee \alpha) / \alpha$ is a semilattice congruence on $S / \alpha$ if and only if $\beta \vee \alpha$ is a semilattice congruence on $S$. The least semilattice congruence on $S$ containing $\alpha$ is $\alpha \vee \lambda(S)$. Thus $\lambda(S / \alpha)=(\lambda(S) \vee \alpha) / \alpha$.

(ii) Let $\beta \in L(S)$ and $\beta \supseteqq \alpha$; then the congruence $\beta / \alpha$ on $S / \alpha$ is idempotent-separating if and only if $\beta|E=\alpha| E$. By definition, the largest congruence coinciding with $\alpha$ on $E$ is $\mu_{\alpha \mid E}$; so $\mu(S / \alpha)=$ $\mu_{\alpha \mid E} / \alpha$.

(iii) Let $\beta \in L(S)$; then, by the same argument as given in (i), $(\beta \vee \alpha) / \alpha$ is a group congruence of $S / \alpha$ if and only if $\beta \vee \alpha$ is a group congruence on $S$. Since the least group congruence on $S$ containing $\alpha$ is $\alpha \vee \sigma(S), \sigma(S / \alpha)=(\sigma(S) \vee \alpha) / \alpha$.

(iv) If $\beta \in L(S)$ and $\beta \supseteqq \alpha$, then the congruence $\beta / \alpha$ on $S / \alpha$ will be idempotent-determined if and only if $\operatorname{ker} \beta=\operatorname{ker} \alpha$. By definition, the largest congruence on $S$ having the same kernel as $\alpha$ is $\tau_{\operatorname{ker} \alpha}$; so $\tau(S / \alpha)=\tau_{\text {ker } \alpha} / \alpha$.

REMARK 3.7. We can now demonstrate that the map $\alpha \mapsto \operatorname{ker} \alpha$ of Theorem 3.4 is not normally join-preserving. We show in the next paragraph that a consequence of the contrary assumption is that any inverse semigroup $S$ having $\tau(S)=\iota$ must be a group. This conclusion is false: $\mathscr{F}_{X}$ (with $X$ any set) is an example of an inverse semigroup which has $\tau=\iota$ but is not a group.

Assume that the map of Theorem 3.4 is join-preserving and let $R$ denote any normal, inverse subsemigroup of $R$. From Theorem 3.3, $R=\operatorname{ker} \lambda_{R}=\operatorname{ker} \tau_{R}$, so by our assumption, $\operatorname{ker} \tau_{R}=\operatorname{ker}\left(\tau_{R} \vee \iota\right)=$ $\operatorname{ker}\left(\tau_{R} \vee \lambda_{E}\right)=\operatorname{ker} \tau_{R} \vee \operatorname{ker} \lambda_{E}=R \vee E=\operatorname{ker} \tau_{R} \vee \operatorname{ker} \tau_{E}=\operatorname{ker}\left(\tau_{R} \vee \tau\right)$. By definition, $\tau_{R}$ is the largest congruence with kernel $R$. It follows 
that $\tau_{R}=\tau_{R} \vee \tau$; whence $\tau \leqq \tau_{R}$. Suppose that $\tau(S)=\iota(S)$. In example (6) of $\S 2.4$, we saw that every inverse semigroup is an idempotent-separating homomorphic image of some $P$-semigroup; so there exists a $P$-semigroup $P$ and an idempotent-separating congruence $\alpha$ on $P$ such that $P / \alpha \cong S$ (suppose, for convenience, that $S=P / \alpha$ ). Proposition 3.6 implies that $\iota(S)(=\tau(S))=\tau(P / \alpha)=\tau_{\text {ker } \alpha} / \alpha$. So, by Lemma 3.5, it follows that $\alpha=\tau_{\text {ker } \alpha}$ and hence, because $\tau(P) \subseteq \tau_{\text {ker } a}$, that $\tau(P) \subseteq \alpha$. Since $\tau=\sigma$ on any $P$-semigroup, $\sigma(P) \subseteq \alpha$ also. By (iii) of Proposition 3.6, $\sigma(S)=(\sigma(P) \vee \alpha) / \alpha=\iota(S)$. Thus $S$ must be a group. This proves the assertions in the last paragraph.

THEOREM 3.8. Each congruence $\alpha$ on an inverse semigroups $S$ is uniquely determined by its kernel and its restriction to idempotents. In fact, $\alpha=\lambda_{\text {ker } \alpha} \vee \sigma_{\alpha{ }^{\prime} E}=\tau_{\text {ker } \alpha} \cap \mu_{\alpha \mid E}$.

Proof. Since $\lambda_{\text {ker } \alpha} \leqq \alpha \cong \tau_{\text {ker } \alpha}$, we deduce from Proposition 3.6 that $\alpha / \lambda_{\text {ker } \alpha}$ must be idempotent-determined on $S / \lambda_{\text {ker } \alpha}$. Proposition 2.5 demands that $\alpha / \lambda_{\text {ker } \alpha}$ be the least congruence with restriction $\left(\alpha / \lambda_{\operatorname{ker} \alpha}\right) \mid E\left(S / \lambda_{\operatorname{ker} \alpha}\right)$ to the idempotents of $S / \lambda_{\operatorname{ker} \alpha}$; so no other idempotent-determined congruence on $S / \lambda_{\text {ker a }}$ can have the same restriction to idempotents as $\alpha / \lambda_{\operatorname{ker} \alpha}$ does. Since the map of Lemma 3.5 is an isomorphism, the above argument ensures that no congruence on $S$ with kernel ker $\alpha$ can also have the same restriction to $E$ as $\alpha$ does. The equations given are now obvious: the congruence $\lambda_{\text {ker } \alpha} \vee \sigma_{\alpha \mid E}$ is contained in $\alpha$ and must have both the same kernel and restriction to idempotents as $\alpha$; the other equality is proved similarly.

Scheiblich [15, Theorem 2.1] gave a nice characterization for congruences in these terms. Suppose $\operatorname{ker} \alpha=R$ and $\alpha \mid E=\pi$. He showed that

$$
\alpha=\left\{(x, y) \in S \times S: x y^{-1} \in R \text { and } a^{-1} a \pi b^{-1} b\right\} .
$$

Also, he effectively gave necessary and sufficient conditions which a pair $(R, \pi)$ must satisfy if some congruence $\alpha$, with the above properties, is to exist. We can derive simpler conditions than his, though, from our considerations above.

Proposition 3.9. Let $R$ be a normal inverse subsemigroup of $S$ and let $\pi$ be a normal congruence on $E$. There exists a congruence $\rho$ on $S$ with $\operatorname{ker} \rho=R$ and $\rho \mid E=\pi$ if and only if

(i) $a \in R$ implies $a a^{-1} \pi \alpha^{-1} a$; and

(ii) for any $x, y$ in $S^{1}$ and any $e, f$ in $E, x e y \in R$ and exf together imply that $x f y \in R$. 
Proof. Suppose that $\rho$ is a congruence on $S$ and has the desired properties. Necessarily, $\rho$ is contained in $\mu_{\pi}$; so $R$ is contained in ker $\mu_{\pi}$. It follows that, for any $a \in R, a a^{-1} \mu_{\pi} a$ and $a \mu_{\pi} a^{-1} a$; whence $R$ and $\pi$ satisfy condition (i). Also, $\tau_{R}$ contains $\rho$. This implies that $\tau_{R} \mid \pi$ contains $\pi$; so, whenever $x e y \in R$ and $e \pi f$, we have $e \tau_{R} f$ and, by the definition of $\tau_{R}, x f y$ must belong to $R$. Thus condition (ii) holds.

Conversely, suppose that $R$ and $\pi$ satisfy the given conditions. Let $\rho=\tau_{R} \cap \mu_{\pi}$. It suffices to show that $\operatorname{ker} \rho=R$ and $\rho \mid E=\pi$. Since $\tau_{R}$ contains $\rho, R$ contains $\operatorname{ker} \rho$. For any element $a$ of $R, a a^{-1} \pi a^{-1} a$ (by condition (i)); so, in $S / \sigma_{\pi}, a \sigma_{\pi}$ and $\left(a a^{-1}\right) \sigma_{\pi}$ are $\mathscr{H}$ equivalent and, by [1, Theorem 7.54] (since $R$ normal implies $x^{-1} R x \subseteq R$, for all $x \in S)$, are identified by $\mu\left(S / \sigma_{\pi}\right)$. Hence, since $\mu\left(S / \sigma_{\pi}\right)=\mu_{\pi} / \sigma_{\pi}$ and noting Lemma 3.5, $a$ and $a a^{-1}$ are $\mu_{\pi}$-equivalent. This ensures that $a$ belongs to both $\operatorname{ker} \mu_{\pi}$ and to $\operatorname{ker} \tau_{R}$. It follows that $\operatorname{ker} \rho=R$.

Since $\mu_{\pi}$ contains $\rho, \pi$ contains $\rho \mid E$. For any $(e, f) \in \pi$, condition (ii) ensures that $x e y \in R$ if and only if $x f y \in R$, for any $x, y$ in $S^{1}$. So $(e, f)$ belongs to $\tau_{R}$ and hence to $\rho$. Therefore $\rho \mid E=\pi$ and the proof is complete.

\section{REFERENCES}

1. A.H. Clifford and G.B. Preston, The algebraic theory of semigroups, Volumes I and II (Math. Surveys 7 (I \& II)), Amer. Math. Soc., Providence, Rhode Island, 1961 and 1967.

2. D. G. Green, Extensions of a semilattice by an inverse semigroup, Bull. Austral. Math. Soc., (1) 9 (1973), 21-31.

3. J. M. Howie, The maximum idempotent-separating congruence on an inverse semigroup, Proc. Edinburgh Math. Soc., (2) 14 (1964), 171-179.

4. D. B. McAlister, Groups, semilattices and inverse semigroups, Trans. Amer. Math. Soc., to appear.

5. — Groups, semilattices and inverse semigroups, Proceedings of a Symposium on Inverse Semigroups, Northern Illinois University, 1973.

6. - Groups, semilattices and inverse semigroups II, preprint.

7. R. McFadden and D. B. McAlister, Zig-zag representations and inverse semigroups, Journal of Algebra, to appear.

8. W. D. Munn, A class of irreducible matrix representations of an arbitrary inverse semigroup, Proc. Glasgow Math. Assn., 5 (1961), 41-48.

9. - A certain sublattice of the lattice of congruences on a regular semigroup, Proc. Camb. Phil. Soc., 60 (1964), 385-391.

10. - Fundamental inverse semigroups, Quart. J. Math. Oxford (2), 21 (1970), $157-170$.

11. G. B. Preston, Inverse semigroups, J. London Math., Soc., (1954), 396-403.

12. N. R. Reilly, Free generators in free inverse semigroups, Bull. Austral. Math. Soc., 7 (1972), 407-424.

13. - Inverse semigroups of partial transformations and $\theta$-classes, Pacific J. Math., 41 (1972), 215-235.

14. N. R. Reilly and H. E. Scheiblich, Congruences on regular semigroups, Pacific J. Math., (2) 23 (1967), 349-360.

15. H. E. Scheiblich, Kernels of inverse semigroup homomorphisms, J. Austral. Math. Soc., to appear. 
16. H. E. Scheiblich, Concerning congruences on symmetric inverse semigroups, Czech. Math. J., 6 (1973).

Received February 25, 1974. This research was supported by a Commonwealth Scholarship. The author is indebted to Professors A.H. Clifford and G. B. Preston, and to the referee, for their suggestions.

DaLhoUSIE UNIVERSITY 


\title{
PACIFIC JOURNAL OF MATHEMATICS
}

\author{
EDITORS
}

RICHARD ARENS (Managing Editor)

University of California

Los Angeles, California 90024

R. A. Beaumont

University of Washington

Seattle, Washington 98105
J. DugundJI

Department of Mathematics University of Southern California Los Angeles, California 90007

D. Gilbarg and J. Milgram

Stanford University

Stanford, California 94305

\section{ASSOCIATE EDITORS}

E. F. BECKENBACH

B. H. NeUManN

F. WOLF

K. YosHIDA

\section{SUPPORTING INSTITUTIONS}

UNIVERSITY OF BRITISH COLUMBIA

GALIFORNIA INSTITUTE OF TECHNOLOGY

UNIVERSITY OF CALIFORNIA

MONTANA STATE UNIVERSITY

UNIVERSITY OF NEVADA

NEW MEXICO STATE UNIVERSITY

OREGON STATE UNIVERSITY

UNIVERSITY OF OREGON

OSAKA UNIVERSITY
UNIVERSITY OF SOUTHERN CALIFORNIA

STANFORD UNIVERSITY

UNIVERSITY OF TOKYO

UNIVERSITY OF UTAH

WASHINGTON STATE UNIVERSITY

UNIVERSITY OF WASHINGTON

AMERICAN MATHEMATICAL SOCIETY NAVAL WEAPONS CENTER 


\section{Pacific Journal of Mathematics}

\section{Vol. 57, No. $1 \quad$ January, 1975}

Keith Roy Allen, Dendritic compactification ......................... 1

Daniel D. Anderson, The Krull intersection theorem ..................... 11

George Phillip Barker and David Hilding Carlson, Cones of diagonally dominant

matrices .............................................

David Wilmot Barnette, Generalized combinatorial cells and facet splitting ........

Stefan Bergman, Bounds for distortion in pseudoconformal mappings ............

Nguyên Phuong Các, On bounded solutions of a strongly nonlinear elliptic

equation ...............................................

Philip Throop Church and James Timourian, Maps with 0-dimensional critical

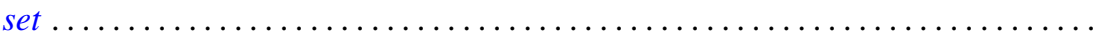

G. Coquet and J. C. Dupin, Sur les convexes ubiquitaires ................. 67

Kandiah Dayanithy, On perturbation of differential operators............... 85

Thomas P. Dence, A Lebesgue decomposition for vector valued additive set

functions .............................................. 91

John Riley Durbin, On locally compact wreath products.................. 99

Allan L. Edelson, The converse to a theorem of Conner and Floyd ............. 109

William Alan Feldman and James Franklin Porter, Compact convergence and the

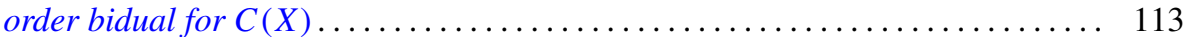

Ralph S. Freese, Ideal lattices of lattices......................... 125

R. Gow, Groups whose irreducible character degrees are ordered by divisibility ... 135

David G. Green, The lattice of congruences on an inverse semigroup ............ 141

John William Green, Completion and semicompletion of Moore spaces .......... 153

David James Hallenbeck, Convex hulls and extreme points of families of starlike and close-to-convex mappings .................................. 167

Israel (Yitzchak) Nathan Herstein, On a theorem of Brauer-Cartan-Hua type ...... 177

Virgil Dwight House, Jr., Countable products of generalized countably compact spaces ............................................ 183

John Sollion Hsia, Spinor norms of local integral rotations. I . . . . . . ......... 199

Hugo Junghenn, Almost periodic compactifications of transformation

semigroups....................................

Shin'ichi Kinoshita, On elementary ideals of projective planes in the 4-sphere and oriented $\Theta$-curves in the 3 -sphere ............................ 217

Ronald Fred Levy, Showering spaces............................ 223

Geoffrey Mason, Two theorems on groups of characteristic 2-type............. 233

Cyril Nasim, An inversion formula for Hankel transform ................... 255

W. P. Novinger, Real parts of uniform algebras on the circle ................ 259

T. Parthasarathy and T. E. S. Raghavan, Equilibria of continuous two-person

games............................................... 265

John Pfaltzgraff and Ted Joe Suffridge, Close-to-starlike holomorphic functions of several variables ....................................... 271

Esther Portnoy, Developable surfaces in hyperbolic space .................. 281

Maxwell Alexander Rosenlicht, Differential extension fields of exponential type . . . 289

Keith William Schrader and James Lewis Thornburg, Sufficient conditions for the

existence of convergent subsequences ........................... 301

Joseph M. Weinstein, Reconstructing colored graphs .................. 307 